\title{
A IMPORTÂNCIA DA ATENÇÃO FARMACÊUTICA EM DROGARIA COMUNITÁRIA: VOLTADA AOS IDOSOS
}

\author{
Luciano Fernandes de Castro ${ }^{1}$ \\ Leonardo Guimarães De Andrade ${ }^{2}$
}

RESUMO: Devido ao número de drogarias existentes no Brasil e a facilidade que a população tem de buscar orientações ao farmacêutico, esse profissional se torna de suma importância para uma manutenção ou melhoria na qualidade de vida. A população idosa que faz uso de vários fármacos e tem dúvidas sobre o uso do mesmo, podem ser orientadas pelo farmacêutico a respeito do uso correto da medicação. Este trabalho visa como a atenção farmacêutica em drogaria é importante para a população, em especial os idosos.

Palavras-chave: Atenção Farmacêutica. Idoso e Drogaria.

ABSTRACT: Due to the number of drugstores that exist in Brazil and the ease that the population has to seek guidance from the pharmacist, this professional becomes extremely important for maintaining or improving the quality of life. The elderly population that uses various medications and has doubts about the same, can be guided by the pharmacist regarding the correct use of the medication. This work aims at how pharmaceutical care in drugstores is important for the population, especially the elderly.

Keywords: Pharmaceutical Care. Elderly and Drugstore.

\section{INTRODUÇÃO}

$\mathrm{Na}$ avaliação da adesão ao tratamento medicamentoso, há existência de um número muito grande de idosos que não costuma utilizar os medicamentos de maneira correta. A justificativa para o tal, se dá por causa das múltiplas patologias e o uso simultâneo de vários medicamentos. (MORSCH et al., 2015).

A exposição a múltiplos fármacos, o uso de mais medicamentos do que está clinicamente indicado, a necessidade clínica indicada está com o consumo de cinco ou mais

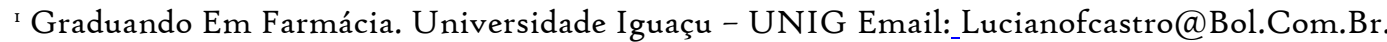

${ }^{2}$ Orientador e Professor do curso de Farmácia- Universidade Iguaçu-Unig.
} 
medicamentos é reconhecida como polifarmácia. Trata-se de uma situação de etiologia multifatorial, maior em indivíduos com doenças crônicas e manifestações clínicas decorrentes do envelhecimento (SALES et al., 2017).

Cabe aos farmacêuticos à responsabilidade de dispensar medicamentos com prescrição médica ou de um dentista ou veterinários, além de poderem recomendar medicamentos de prescrição isenta, estabelecer o perfil farmacoterapêutico do paciente, prestando orientação Farmacêutica e sanando as dúvidas quanto a relação benefício e risco, conservação e utilização destes medicamentos, assim como as prováveis interações medicamentosas dos mesmos. A dispensação, por sua vez, consiste no ato farmacêutico de orientar e fornecer ao usuário de medicamentos, correlatos ou insumos farmacêuticos, podendo ser esta remunerada ou não (BRASIL, 2014).

Esse alto número de medicamento pode acarretar problemas para a saúde como uma potencialização de efeito adversos ou mesmo uma diminuição da ação dos fármacos. (SILVEIRA et al., 2018).

Pessoas idosas demandam de um cuidado mais específico, uma vez que carecem de uma assistência contínua das doenças crônicas e, ocasionalmente necessitam ser orientados para problemas de saúde que apareçam. Precisam estar esclarecidos a respeito do uso dos medicamentos e de suas enfermidades. A Atenção Farmacêutica na drogaria, contribui de maneira significativa em uma manutenção ou melhora na qualidade de vida dessas pessoas. (BORTOLON et al., 2017).

A área de Atenção Farmacêutica para idosos é de suma importância, afinal, essa ação, tem como propósito, minimizar a quantidade de hospitalizações e óbitos pertinentes aos danos das doenças crônicas, nas questões relacionadas a adesão farmacoterapêutica, nos riscos da automedicação e assessorar o prescritor na escolha de medicamento adequado, isto é, contribuir de modo direto reduzindo as adversidades para continuidade da terapia garantindo uma melhora de vida. (CARVALHO; SENA, 2017).

A Atenção Farmacêutica facilita a entrada do farmacêutico na equipe multidisciplinar, agrega valores à profissão onde na maioria das vezes é observada com surpresa por parte das pessoas ao visualizarem as novas atividades dos profissionais Farmacêuticos. Já que o trabalho dos Farmacêuticos poderá trazer benefícios aos pacientes, observa-se que a 
implantação de serviços farmacêuticos soma positivamente, trazendo bons resultados junto a população assistida (BRASIL, 20I6).

De acordo com o CFF, temos mais de 87 mil farmácias e drogarias privadas no Brasil ( $\mathrm{CFF}$ - 2018), esse número torna o farmacêutico um profissional que se encontra em um local de trabalho estratégico, ou seja, a população tem acesso a esse profissional de maneira ampla, recorrendo ao farmacêutico, de maneira acessível. (OLIVEIRA et al., 2017).

Aprimorar a habilidade de comunicação é essencial para a atenção farmacêutica com o intuito de conseguir resultados satisfatório com as intervenções sugeridas. (BISSON 2017).

A intervenção é importante para prevenir ou resolver um problema que seja capaz de atrapalhar no tratamento de pacientes com medicamentos, realizando acompanhamento farmacoterapêutico. Na drogaria o Farmacêutico é responsável por cuidar das dificuldades dos pacientes relacionadas aos medicamentos, localizando e eliminando problemas relacionados aos medicamentos, como as reações adversas. (CRF-CE 2018).

A PNM - Política Nacional de Medicamentos discorre sobre elementos importantes, dentre eles a promoção do uso de forma racional de medicamentos e a garantia da eficácia, assim como a segurança e qualidade para o uso de medicamentos através do ciclo em que consiste a Assistência Farmacêutica. Desta forma, o farmacêutico dispõe-se de um papel de destaque nas etapas de gestão desse ciclo de assistência, dado que é ele quem está em primeiro contato com o paciente, e para isso ele precisa de habilidades e competências técnicas para que consiga prover a orientação correta para o sucesso da farmacoterapia influenciando o processo de saúde e doença do paciente (FLORENTINO, 20I6).

Outro fator importante com relação aos idosos é o fato da idade avançada contribuir para erro de autoadministrações medicamentosas por meio da confusão causada pela quantidade excessiva de medicamentos diários, a visão prejudicada que faz confundir as caixas dos fármacos semelhantes, destreza manual prejudicada e distúrbios cognitivos (LUPPI, CARVALHO, 2005).

Entre os erros que ocorrem na administração de medicamentos, destacam-se a escolha do medicamento incorreto, uso incorreto do medicamento, caracterizado por superdosagem, dose sub- terapêuticos e horários de administração inadequados. Outros erros comuns são a automedicação e interações medicamentosas (PERETTA; CICCIA, 20oo). 
As pessoas acima de 65 anos, possuem doenças crônicas como, as doenças osteoarticulares, a hipertensão arterial sistêmica, diabetes, entre outras, fazendo com que dependam de tratamento medicamentoso prolongado e contínuo e dificuldades fisiológicas devido a alterações da massa corporal, com diminuição da quantidade de água, redução das taxas de excreção renal entre outras e isso aparece devido à idade avançada e podem precisar fazer uso de vários fármacos o que leva a causa da automedicação nesta população e por isso torna o grupo mais medicalizado. (BORTOLON; KARNIKOWSKI; ASSIS, 2007; SÁ; BARROS; SÁ, 2007; CASCAES; FALCHETTI; GALATO, 2008).

Existem alguns costumes entre os idosos que interferem no efeito do tratamento farmacológico como armazenar os medicamentos dentro de armários da cozinha, banheiros ou em locais indevidos e a falta de hábitos ou mesmo pela dificuldade em verificar a data de validade e ainda uma prática muito comum entre os idosos, é o costume de repartir o comprimido e armazenar a outra parte para tomar depois, guardar os fármacos fora da embalagem de origem, manipular os fármacos com mãos sujas, entre outros. (ALVES; ALVES; PARTATA, 20I0).

Segundo Carvalho et al. (2012) a farmacologia para os idosos necessita de muito cuidado. Com a idade, a massa muscular e a água corporal diminuem, podendo comprometer o metabolismo hepático, os mecanismos homeostáticos, a filtração e excreção renal, assim dificultando a eliminação de metabólitos, acúmulo de substâncias tóxicas, aumentando as reações adversas.

\section{OBJETIVOS GERAL}

Este trabalho tem por objetivo evidenciar a importância que a um profissional Farmacêutico faz ao orientar a população a cerca de um medicamento, quanto ao seu uso correto, interações e como deve ser guardado.

Demonstrar como a Atenção Farmacêutica na drogaria comunitária, com as pessoas idosas, é importante para uma melhor qualidade de vida.

\section{OBJETIVOS ESPECÍFICOS}

- Descrever a importância do farmacêutico na drogaria. 
- Evidenciar melhoria na qualidade de vida.

- Apontar as dificuldades dos idosos com o uso de medicamentos simultâneos.

- Mostrar como a população tem um acesso facilitado a orientação farmacêutica por meio da drogaria.

\section{METODOLOGIA}

A metodologia empregada neste trabalho foi a de pesquisa bibliográfica. Realizou-se uma pesquisa sobre como a Atenção Farmacêutica na drogaria comunitária pode ser importante para contribuição da saúde na população idosa. Foi utilizado, como fonte de pesquisa, artigos publicados em periódicos e tendo como base de dados o Scielo e Google acadêmico, utilizando as palavras-chave: Atenção Farmacêutica, idoso e drogaria, no período entre 2019 à 2021.

\section{JUSTIFICATIVA}

O presente artigo tem por intuito ressaltar a importância do Farmacêutico nas drogarias. Mostrando como esse profissional pode se portar em relação a melhoria da qualidade de vida de seus pacientes. É apontado que os idosos fazem uso de vários medicamentos ao mesmo tempo e o Farmacêutico é capacitado para intervir, quando necessário, em uma possível diminuição de efeito adversos provocados pelo uso de vários medicamentos. O farmacêutico pode contribuir de maneira significativa com o médico através da otimização da farmacoterapia, garantindo assim, segurança e efetividade no tratamento farmacológico.

Não são objetivos da prática da Atenção Farmacêutica o diagnóstico de condições de saúde ou a prescrição de medicamentos, os quais são de responsabilidades dos profissionais médicos ou dentistas. Ao contrário, as ações Farmacêuticas devem ser direcionadas especificamente à resolução de problemas relacionados aos medicamentos, no contexto de uma relação terapêutica construída com o usuário. Desse modo a interação entre 
Farmacêuticos e prescritores deve ser realizada buscando o benefício do paciente numa atuação conjunta, que pode contribuir também para o fortalecimento da equipe de saúde.

\section{ATENÇÃO FARMACÊUTICA AOS IDOSOS}

Carrol e Brue (199I) e Couto (2000) relatam que o envelhecimento passa a ser caracterizado pela incapacidade progressiva de o organismo adaptar-se às condições variáveis do seu ambiente. Os mecanismos implícitos neste episódio apresentam as seguintes características: são progressivos, nocivos, irreversíveis e geralmente comuns a inúmeros organismos, sendo semelhantes na mesma espécie.

Os idosos chegam a constituir 50\% dos multiusuários de medicamentos. Por conseguinte, é comum encontrar, em suas prescrições, dosagens e indicações inadequadas, interações medicamentosas, associações e redundâncias (uso de fármacos pertencentes a uma mesma classe terapêutica) e medicamentos sem valor terapêutico. Tais fatores podem gerar reações adversas aos medicamentos, algumas delas graves e fatais (OLIVEIRA, I994; SCHRADER et al., 1996; MOSEGUI et al., 1999).

O uso de medicamentos por idosos tem uma linha tênue entre o risco e o benefício, ou seja, a elevada utilização de medicamentos pode afetar a qualidade de vida do idoso, por outro lado, são os mesmos que, em sua maioria, ajudam a prolongar a vida. Logo, o problema não pode ser atribuído ao consumo do medicamento, mas sim na irracionalidade de seu uso, que expõe o geronto a riscos potenciais (LE SAGE, 1991; TAPIA-CONYER et al., 1996).

Em diferentes estudos sobre o consumo de medicamentos, por idosos, verificou-se de um grande número de especialidades farmacêuticas, com prevalência do uso de analgésicos, antiinflamatórios e psicotrópicos (MOSEGUI et al., 1999; CASTRO, 200I).

O uso de medicamentos em idosos é elevado e pode afetar a qualidade de vida destes quando da irracionalidade de sua utilização, sujeitando-o a riscos potenciais, sendo que estes podem ser elevados pelas alterações nos aspectos farmacocinéticos e farmacodinâmicos decorrentes de modificações fisiológicas próprios da idade (DUTHE, et al., 2002).

Essas situações são agravadas pela polimedicação, que leva a riscos de interações medicamentosas e reações adversas, fora as associações de remédios que muitas vezes não apresentam racionalidade terapêutica (ANDRADE, et al., 2004). 
Uma não adesão ao tratamento é a principal responsável pelas falhas no tratamento e por agravos no processo patológico. Apresentando assim, maiores custos a saúde pública devido à amplificação nos números de casos de intoxicação e internações hospitalares (CARVALHO, et al., 2012).

\section{CONCLUSÃO}

Com a problemática dos idosos utilizarem vários medicamentos simultaneamente, além de demandarem de um cuidado mais específico por causa das doenças crônicas, fica evidente como a atenção farmacêutica na drogaria pode contribuir para uma melhora ou manutenção de vida desse grupo de pessoas.

Visto que os idosos são mais propensos a terem problemas crônicos, eles acabam utilizando mais medicamentos do que os grupos mais jovens no geral. A utilização de medicamentos mútuos pode vir a gerar duvidas, tornando o farmacêutico indispensável na drogaria para o auxílio ideal do uso do medicamento de acordo com a prescrição médica.

$\mathrm{Na}$ drogaria o farmacêutico pode atuar de diversas formas, desde a orientação sobre os riscos da automedicação ou até mesmo ajudando o médico reduzindo as adversidades provocadas, por exemplo, pelo uso simultâneo de vários medicamentos.

Para os idosos com dificuldade do receituário com vários medicamentos o farmacêutico pode simplificar de uma maneira que fique clara para que esse idoso compreenda. E a dúvida, muita das vezes é tirada antes da próxima consulta médica, podendo assim, o idoso ter um tratamento adequado. Sem o profissional farmacêutico e com uma consulta marcada dali a meses, o tratamento poderia se tornar ineficaz e até colocar a saúde ou a vida do idoso em risco.

Com as várias farmácias espalhadas pelos Brasil, o farmacêutico se torna mais acessível para população, ajudando diversas pessoas diariamente. Atuando na atenção farmacêutica, o farmacêutico busca o sucesso terapêutico orientando o paciente idoso com sua terapia. 


\section{REFERÊNCIAS}

ALVES, A. J.; ALVES, L. K.; PARTATA, A. K. Atuação do farmacêutico na promoção e restauração da saúde de pacientes idosos que fazem uso de polimedicação. Revista cientifica do ITPAC, v.3, n.3, p.4-23, abr. 2010. Disponível em:. Acesso em: o5 jun. 2012.

ANDRADE, M. A.; SILVA, M. V. S.; FREITAS, O. Assistência Farmacêutica como Estratégia para o Uso Racional de Medicamentos em Idosos. Semina: Ciências Biológicas e da Saúde. V. 25, n. I, p.56-6o, 2004.

BISSON, M. P. Farmácia clínica \& atenção farmacêutica. 2. ed. São Paulo: Manole, 2017.

BORTOLON et al. Automedicação versus indicação farmacêutica: o profissional de farmácia na atenção primária à saúde do idoso. Revista APS, v.ı, n.2, 2017, p. 200-209.

BORTOLON, P. C.; KARNIKOWSKI, M. O. G; ASSIS, M. Automedicação versus indicação farmacêutica: o profissional de farmácia na atenção primária à saúde do idoso. Revista APS, v.1o, n.2, p. 200-209, jul./dez. 2007. Disponível em: Acesso em: 13 set. 2011.

BRASIL. CASA CIVIL. Lei n.ำ 13.021, de 8 de agosto de 2014, que dispões sobre o exercício e as atividades farmacêuticas.

BRASIL. CONSELHO FEDERAL DE FARMÁCIA. Implantação do serviço de acompanhamento farmacoterapêutico em unidade de atenção primária à saúde de Betim: experiência do Projeto Dia a Dia. Relato da Experiência, Brasília-DF, v. 4, n. 4, p.40-48, 2016.

CARROL, M.; BRUE, L. J. Enfermagem para idosos: guia prático. São Paulo: Andrei, I99ı.

CARVAlHO, A. L. M.; LEOPOLDINO, R. W. D.; SIlVA, J. E. G.; CUNHA, C. P. Adesão ao tratamento medicamentoso em usuários cadastrados no programa hiperdia no município de Teresina (PI). Revista ciências e saúde coletiva I7(7): 1885-1892 2012. 
CARVALHO, J. S.; SENA, C. F. A. Problemas relacionados à manutenção do tratamento medicamentoso em pacientes idosos e as contribuições da atenção farmacêutica. Revista Brasileira de Ciências da Vida, v. 5, n. I, 2017.

CARVALHO, M.H.C. I Diretriz brasileira de diagnóstico e tratamento da síndrome metabólica. Arquivos Brasileiros de Cardiologia, 84p. 2005.

CASTRO, L. L. C. Fundamentos de Farmacoepidemiologia: uma introdução ao estudo da Farmacoepidemiologia. Campo Grande: Grupuram, 20oI.

Conselho Federal de Farmácia - CE.

COUTO, L. B. Aspectos farmacológicos do uso de medicamentos em idosos. Revista Racine, São Paulo, v.56, maio/jun., p.58-62, 2000.

DA SILVEIRA, Priscila Assis; SILVA, Samuel Campos; ROCHA, Karine Siqueira Cabral.

Prevalência da Polifarmácia nos Idosos de uma Unidade Básica de Saúde no Estado de Minas Gerais. Revista de Atenção à Saúde (antiga Rev. Bras. Ciên. Saúde), v. I6, n. 58, 2019.

DUThE, E. H.; KATZ, P. R. Geriatria Prática. 3. Ed. Editora Revinter. Rio de Janeiro, pag.582. 2002.

FLORENTINO, M. The education pharmacist continuing in a private pharmacy network in Florianópolis /SC.2016.

LE SAGE J. Polipharmacy in geriatric patients. Nursing Clinics of North America, Philadelphia, v.26, p.273-290, 1991.

LUPPI, G.; CARVALHO, M.F.C. Atenção farmacêutica em pacientes geriátricos: uma experiência no Centro de Referência do Idoso. Cadernos da Faculdades Integradas São Camilo, v.II, n.4, p. 90-96, 2005. 
MORSCH, Lisoni Muller et al. Complexidade da farmacoterapia em idosos atendidos em uma farmácia básica no Sul do Brasil. Ver. Infarma Ciências Farmacêuticas, v. 4, n. 27, p. 239-247, 2015 .

MOSEGUI, G. B.G.; ROZENFELD, S.; VERAS, R. P.; VIANNA, C. M. M. Avaliação da qualidade do uso de medicamentos em idosos. Revista de Saúde Pública, São Paulo, v.35, n.5, p.437-444, 1999 .

MOSEGUI, G. B.G.; ROZENFELD, S.; VERAS, R. P.; VIANNA, C. M. M. Avaliação da qualidade do uso de medicamentos em idosos. Revista de Saúde Pública, São Paulo, v.35, n.5, p.437-444, 1999 .

OLIVEIRA, G. G. A base farmacocinética da abordagem terapêutica nos idosos. Folha Médica, Rio de Janeiro, v.io9, n.2, p.77-8I, 1994.

OLIVEIRA, Naira Villas Boas Vidal de et al. Atuação profissional dos farmacêuticos no Brasil: perfil sociodemográfico e dinâmica de trabalho em farmácias e drogarias privadas. Saúde e Sociedade, v. 26, p. IIO5-II2I, 2017. DOI: I0.1590/so104-I2902017000002.

PERETTA, M.; CICCIA, G. Reengenharia farmacêutica: guia para implementar a atenção farmacêutica. Brasília, 2000.

SALES, A. S.; Sales, M. G. S.; Casotti, C. A. Perfil farmacoterapêutico e fatores associados à polifarmácia entre idosos de Aiquara, Bahia, em 2014. Epidemiologia e Serviços de Saúde, v. 26, p. 121-132, 2017.

SCHRADER, S. L.; DRESSING, B.; BLUE, R.; JENSEN, G.; MILLER, D.; ZAWADA, E. T. The medication reduction project: combating polypharmacy in South Dakota elders through community-based interventions. South Dakota Journal of nmedicine, Sioux Falls, v.49, n.12, p.441-8, 1996. 